\title{
Performance of MPGD-based TPC Prototypes for the Linear Collider Experiment
}

M. Kobayashi ${ }^{\text {a }}$, T. Araki ${ }^{\text {b }}$, D.C. Arogancia ${ }^{\text {c }}$, A.M. Bacala ${ }^{\mathrm{c}}$, K. Boudjemline $^{\mathrm{d}}$, D. Burke ${ }^{e}$, P. Colas ${ }^{\text {e }}$, M. Dixit d,f, K. Fujii ${ }^{a}$, H. Fujishima ${ }^{b}$, A. Giganon ${ }^{e}$, I. Giomataris ${ }^{\text {e }}$, H.C. Gooc Jr ${ }^{\mathrm{c}}$, M. Habu ${ }^{\mathrm{g}}$, T. Higashi ${ }^{\mathrm{b}}$, K. Ikematsu ${ }^{\text {h}}$, K. Kadomatsu ${ }^{\text {b }}$, Y. Kato ${ }^{\text {i, H. Huroiwa }}{ }^{\text {a }}$, V. Lepeltier ${ }^{j}$, T. Matsuda ${ }^{\text {a }}$, S. Matsushita ${ }^{g}$, K. Nakamura ${ }^{g}$, O. Nitoh ${ }^{g}$, J. Pouthas ${ }^{k}$, R.L. Reserva ${ }^{\text {c }}$, P. Rosier ${ }^{k}$, R.D. Settles ${ }^{\text {l, }}$ A. Sugiyama ${ }^{\text {b }}$, T. Takahashi ${ }^{\mathrm{m}}$, Y. Tanaka ${ }^{\mathrm{b}}$, T. Watanabe ${ }^{\mathrm{n}}$, A. Yamaguchi ${ }^{\circ}$, T. Yamamoto $^{\mathrm{b}}$, H. Yamaoka $^{\text {a }}$, T. Zerguerras $^{\mathrm{k}}$

${ }^{a}$ High Energy Accelerator Research Organization (KEK), Tsukuba, 305-0801, Japan

${ }^{b}$ Saga University, Saga, 840-8502, Japan

${ }^{c}$ MSU-Iligan Institute of Technology, Iligan, Philippines

${ }^{d}$ Carleton University, Ottawa, ON, K1S 5B6, Canada

${ }^{e}$ DAPNIA, CEA Saclay, 91191 Gif sur Yvette, France

${ }^{f}$ TRIUMF, Vancouver, BC, V6T 2A3, Canada

${ }^{g}$ Tokyo University of Agriculture and Technology, Koganei, 184-8588, Japan

${ }^{h}$ DESY, DE-22607 Hamburg, Germany

${ }^{i}$ Kinki University, Higashi-Osaka, 577-8502, Japan

${ }^{j} L A L$, IN2P3-CNRS, et Universite Paris-Sud 11, F91898 Orsay, France

${ }^{k}$ IPN, IN2P3-CNRS, et Universite Paris-Sud 11, F91406 Orsay, France

${ }^{l}$ Max Planck Institute for Physics, DE-80805 Munich, Germany

${ }^{m}$ Hiroshima University, Higashi-Hiroshima, 739-8530, Japan

${ }^{n}$ Kogakuin University, Hachioji, 192-0015, Japan

${ }^{\circ}$ University of Tsukuba, Tsukuba, 305-8573, Japan

\begin{abstract}
We conducted a series of beam tests of prototype TPCs for the International Linear Collider (ILC) experiment, equipped with an MWPC, a MicroMEGAS, or GEMs as a readout device. The prototype operated successfully in a test beam at KEK under an axial magnetic field of up to $1 \mathrm{~T}$. The analysis of data is now in progress and some of the preliminary results obtained with GEMs and MicroMEGAS are presented along with our interpretation. Also given is the extrapolation of the obtained spatial resolution to that of a large TPC expected as the central tracker of the ILC experiment.
\end{abstract}

PACS: 29.40.Cs; 29.40.Gx

Keywords: TPC; GEM; MicroMEGAS; ILC; Spatial Resolution 


\section{Introduction}

One of the major physics goals of the future linear collider experiment is to study properties of the Higgs boson, which is expected to be well within the reach of the center-of-mass energy of the machine [1][2]. This goal demands unprecedented high performance of each detector component. For example, the central tracker is required to have good spatial resolution, high two-track resolving power, and high momentum resolution, for precise reconstruction of hard muons and each of the charged particle tracks in dense jets.

A time projection chamber (TPC) is a strong candidate for the central tracker of the experiment since it can cover a large volume with a small material budget while maintaining high three dimensional granularity. If micro-pattern gas detectors (MPGDs: micro-mesh gaseous structure (MicroMEGAS) [3], gas electron multiplier (GEM) [4] etc.) are employed for the detection devices of the TPC, instead of conventional multi-wire proportional chambers (MWPCs), one can expect higher granularity and better spatial resolution with a smaller or negligible $E \times B$ effect at the entrance to the detection plane. Furthermore, the MPGDs have inherently smaller positive ion back flow rate than the MWPCs. We therefore constructed a small prototype TPC with a replaceable readout device (MWPC, MicroMEGAS or triple GEM) and have conducted a series of beam tests at KEK in order to study its performance, especially its spatial resolution under an axial magnetic field.

We begin with brief descriptions of the prototype TPC and the experimental setup. Next, some preliminary results are presented along with our interpretation, in which special emphasis is placed on an analytic expression of the spatial resolution. Finally, the spatial resolution of the ILC-TPC is estimated from the measurement with the prototypes.

\section{$2 \quad$ Experimental setup}

A photograph of the prototype is shown in Fig. 1. It consists of a field cage and an easily

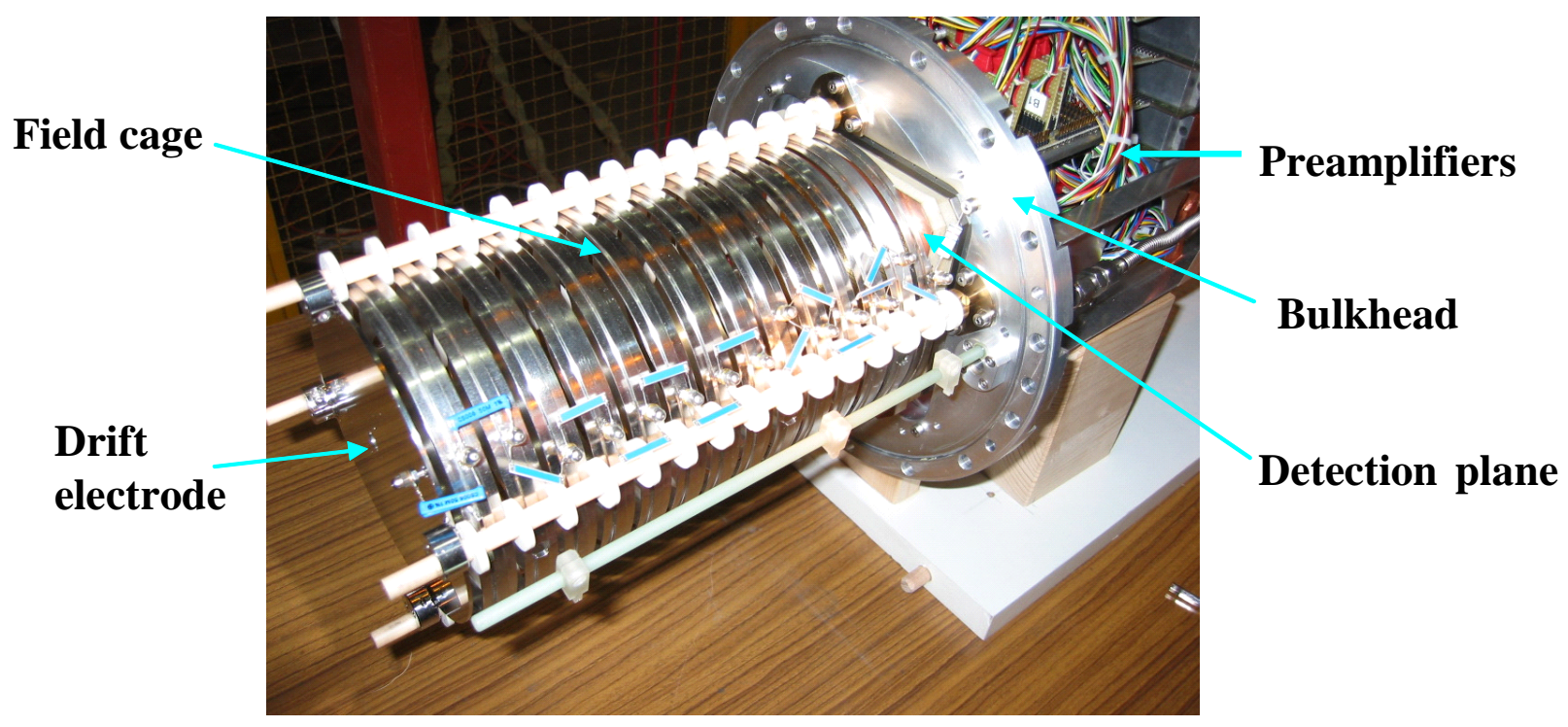

Figure 1: Photograph of the prototype just before installation into the gas vessel.

replaceable gas amplification device attached to one end of the field cage. Gas amplified electrons are detected by a pad plane at ground potential placed right behind the amplification device. A drift electrode is attached to the other end of the field cage. The maximum drift length is about $260 \mathrm{~mm}$. 
The pad plane, with an effective area of $\sim 75 \times 75(100 \times 100) \mathrm{mm}^{2}$, has $12(16)$ pad rows at a pitch of $6.3 \mathrm{~mm}$, each consisting of $2 \times 6(1.17 \times 6) \mathrm{mm}^{2}$ rectangular pads arranged at a pitch, denoted hereafter as $w$, of $2.3(1.27) \mathrm{mm}$ when combined with MicroMEGAS (GEMs). The neighboring pad rows are staggered by half a pad pitch in the case of the GEM readout. Pad signals are fed to charge sensitive preamplifiers located on the outer surface of the bulkhead of the gas vessel behind the pad plane. The amplified signals are sent to shaper amplifiers in the counting room via twisted pair cables, and then processed by $12.5 \mathrm{MHz}$ digitizers. ${ }^{1}$

The mesh of MicroMEGAS, made of $5 \mu \mathrm{m}$ thick copper, has $35 \mu \mathrm{m}$ holes spaced at intervals of $61 \mu \mathrm{m}$. The distance between the mesh and the pad plane is maintained at $50 \mu \mathrm{m}$ by kapton pillars arranged in-between. The typical gain is about 3650 at a mesh potential of $-320 \mathrm{~V}$. The triple GEM, CERN standard, has two $1.5 \mathrm{~mm}$ transfer gaps and a $1 \mathrm{~mm}$ induction gap. The transfer and induction fields are about $2 \mathrm{kV} / \mathrm{cm}$ and $3 \mathrm{kV} / \mathrm{cm}$, respectively. The total effective gain in a $\mathrm{P} 5$ gas (TDR gas) is about 3000 with $335 \mathrm{~V}(340 \mathrm{~V})$ applied across each GEM foil.

The used chamber gases are Ar-isobutane (5\%) for MicroMEGAS, and a TDR gas (Ar-methane (5\%)-carbon dioxide (2\%)) or a P5 gas (Ar-methane (5\%)) for GEMs, at atmospheric pressure and room temperature. The gas pressure and the ambient temperature are continuously monitored since they are not controlled actively. The drift-field strengths $(E)$ are 220, 235 and $100 \mathrm{~V} / \mathrm{cm}$, respectively for Ar-isobutane, TDR gas and Ar-methane.

The prototype TPC is placed in the uniform field region of a superconducting solenoid without return yoke, having a bore diameter of $850 \mathrm{~mm}$, an effective length of $1000 \mathrm{~mm}$, and a maximum field strength of $1.2 \mathrm{~T}$. The prototype was then subjected to the beam, mostly $4 \mathrm{GeV} / c$ pions, at the $\pi 2$ test beam facility of the KEK proton synchrotron.

\section{Preliminary results}

In this section we show some preliminary results of the analysis up to now, only for the data taken with an axial magnetic field $(B)$ of $1 \mathrm{~T}$ and with tracks perpendicular to the pad rows. ${ }^{2}$ The results of the analytic calculations are used or presented here without descriptions. Interested readers are therefore suggested to refer to the appendix of Ref. [5], where the analytic approach is briefly summarized and an analytic formula for the spatial resolution is given.

The observed pad responses for different drift distances $(z)$ are shown in Fig. 2 (a) while the widths of the distributions are plotted as a function of the drift distance in Fig. 2 (b). Figs. 3 (a) and
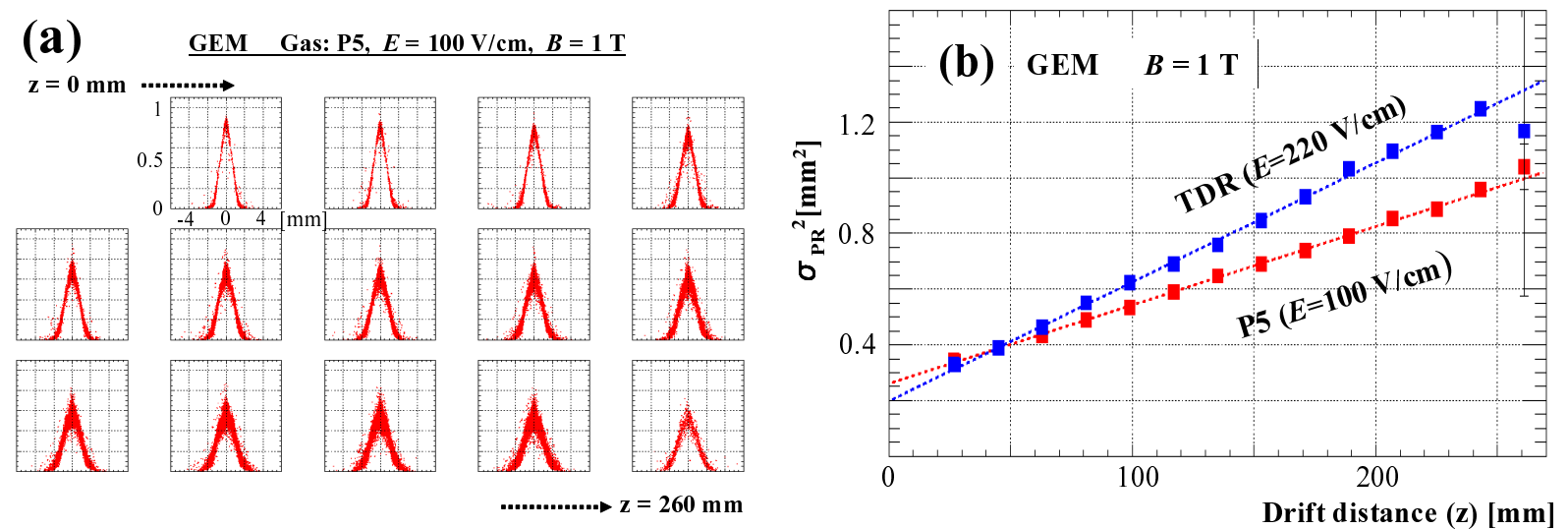

Figure 2: (a) Pad responses for different drift distances. (b) Pad-response width squared $\left(\sigma_{\mathrm{PR}}^{2}\right)$ vs. drift distance $(z)$. The width of the pad response is parametrized as $\sigma_{\mathrm{PR}}^{2}=\sigma_{\mathrm{PR} 0}^{2}+D^{2} \cdot z$, with $D$ being the diffusion constant.

\footnotetext{
${ }^{1}$ The ALEPH TPC electronics was used without modification.

${ }^{2}$ It should be pointed out here that the pad rows were effectively staggered even in the case of the MicroMEGAS readout since the beam had a small but finite average angle of $\sim 3^{\circ}$ with respect to the pad-row normal.
} 
(b) show the spatial resolution in the pad row direction, obtained with a charge centroid method, plotted against the drift distance $(z)$, respectively for the MicroMEGAS and the triple GEM readout, along with the results of analytic calculations. In the calculation the pad response function (PRF) was assumed to be a $\delta$ function for the MicroMEGAS and a Gaussian for the GEMs. ${ }^{3}$
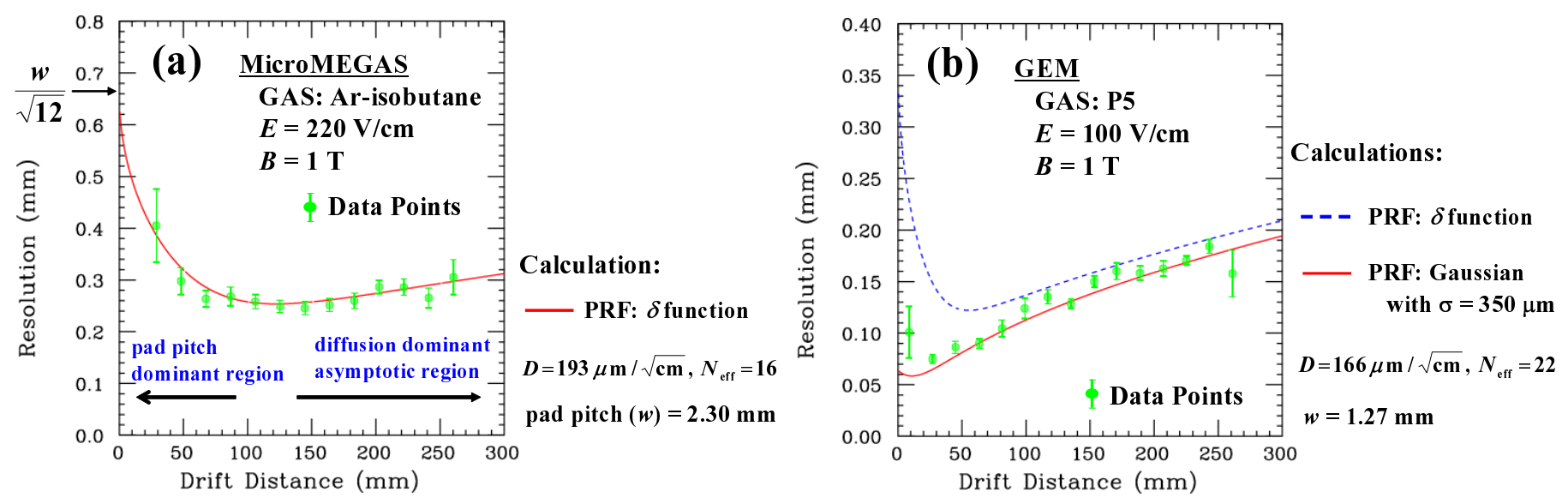

Figure 3: (a) Spatial resolution vs. $z$ obtained with MicroMEGAS, $w=2.3 \mathrm{~mm}$, gas: Ar-isobutane (5\%). (b) Spatial resolution vs. $z$ obtained with GEMs, $w=1.27 \mathrm{~mm}$, gas: Ar-methane (5\%).

The obtained behavior of the pad response, and the spatial resolution at long drift distances ${ }^{4}$ are summarized in Table 1 and compared with expectations. The comparisons show

i $\sigma_{\mathrm{PR} 0}$ is in reasonable agreement with the expectation $\left(\sqrt{w^{2} / 12+\sigma_{\mathrm{PRF}}^{2}}\right)$ if the contribution of $\sigma_{\mathrm{PRF}}$ is taken into account (in the case of GEMs);

ii The values of the diffusion constant $(D)$ are comparable to those given by the simulation (MAGBOLTZ [7]);

iii $\sigma_{\mathrm{X} 0}$ is consistent with the expectation $\left(w / \sqrt{12 \cdot N_{\mathrm{eff}}}\right)$ for the MicroMEGAS, and better than this for the GEMs because of the sizable charge spread in the transfer and induction gaps;

iv $N_{\text {eff }}(16-22)$ is significantly smaller than the average number of drift electrons per pad row $(\sim 71)[6]$.

\footnotetext{
${ }^{3} \mathrm{PRF}$ is the avalanche charge spread on the pad plane for a single drift electron and should not be confused with the pad response. In the case of MicroMEGAS it is much smaller than the pad pitch (2.3 mm) and is therefore neglected. The width (standard deviation) of the Gaussian PRF for the triple GEM has been determined from the intercept of the pad-response width squared vs. $z$ (Fig. $2(\mathrm{~b})): \sigma_{\mathrm{PR}}^{2}=\sigma_{\mathrm{PR} 0}^{2}+D^{2} \cdot z$ with $\sigma_{\mathrm{PR} 0}^{2}=w^{2} / 12+\sigma_{\mathrm{PRF}}^{2}$, where the pad pitch $w=1.27 \mathrm{~mm}$ and $\sigma_{\mathrm{PR} 0} \sim 511 \mu \mathrm{m}$, yielding $\sim 356 \mu \mathrm{m}$ for $\sigma_{\mathrm{PRF}}$. The value of $\sigma_{\mathrm{PRF}}$ thus obtained is consistent with a simple estimate taking into account only the diffusion in the transfer and induction gaps $(\sim 360 \mu \mathrm{m})$.

${ }^{4}$ The asymptotic behavior of the spatial resolution at long distances (diffusion dominant asymptotic region) is described by $\sigma_{\mathrm{X}}^{2}=\sigma_{\mathrm{X} 0}^{2}+D_{\mathrm{X}}^{2} \cdot z$ with $D_{\mathrm{X}}^{2}=D^{2} / N_{\text {eff }}$, where $D$ is the diffusion constant and $N_{\text {eff }}$ the effective number of electrons [5]. $\quad N_{\text {eff }}$ is determined by the primary ionization statistics and the relative variance of the avalanche fluctuation for a single drift electron [6]. When PRF is a $\delta$ function, $\sigma_{\text {X0 }}^{2}$ is given by $w^{2} /\left(12 \cdot N_{\text {eff }}\right)$.
} 
Table 1. Asymptotic behavior at long drift distances under $B=1 \mathrm{~T}$, (a) for the pad response and (b) for the spatial resolution.

(a) Pad response

\begin{tabular}{|c||c||c|c|}
\hline Detection device & MicroMEGAS & \multicolumn{2}{c|}{ GEM } \\
\hline \hline Gas & Ar-isobutane $(5 \%)$ & TDR & Ar-methane $(5 \%)$ \\
\hline$\sigma_{\text {PR0 }}(\mu \mathrm{m})$ & $758 \pm 91$ & $432 \pm 3$ & $511 \pm 2$ \\
\hline$w / \sqrt{12}(\mu \mathrm{m})$ & 664 & & 367 \\
\hline$D(\mu \mathrm{m} / \sqrt{\mathrm{cm}})$ & $194 \pm 18$ & $213 \pm 1$ & $168 \pm 1$ \\
\hline$D[$ MAGBOLTZ $]$ & 193 & 209 & 166 \\
\hline
\end{tabular}

(b) Spatial resolution

\begin{tabular}{|c||c||c|c|}
\hline \multicolumn{1}{|c||}{ Detection device } & MicroMEGAS & \multicolumn{2}{c|}{ GEM } \\
\hline \hline Gas & Ar-isobutane $(5 \%)$ & TDR & Ar-methane $(5 \%)$ \\
\hline$\sigma_{\mathrm{X} 0}(\mu \mathrm{m})$ & $161 \pm 54$ & $44 \pm 10$ & $42 \pm 17$ \\
\hline$w / \sqrt{12 \cdot N_{\mathrm{eff}}}(\mu \mathrm{m})$ & $166 \pm 42$ & $86 \pm 3$ & $78 \pm 4$ \\
\hline$D / \sqrt{N_{\mathrm{eff}}}(\mu \mathrm{m} / \sqrt{\mathrm{cm}})$ & $48 \pm 12$ & $47 \pm 1$ & $35 \pm 2$ \\
\hline$N_{\mathrm{eff}}$ & $16 \pm 8$ & $20 \pm 1$ & $22 \pm 2$ \\
\hline
\end{tabular}

\section{Comparison with simulation}

In order to confirm the reliability of the analytic calculation, the spatial resolution was estimated by means of a realistic Monte-Carlo simulation as well. ${ }^{5}$ Fig. 4 compares the expected resolutions obtained with the two methods for the triple GEM readout, along with the data points. The MonteCarlo simulation takes into account the primary ionization statistics, diffusion in the drift region, avalanche multiplication and its fluctuation in the GEM holes, and the diffusion in the transfer and induction gaps. The results are almost identical, demonstrating the reliability of both the analytic approach and the Monte-Carlo simulation. The major advantage of the simulation is its applicability to inclined tracks, whereas the analytic formula assumes only tracks perpendicular to the pad row.

\section{Expected spatial resolution of the ILC-TPC}

Calculated spatial resolutions of the ILC-TPC at $B=4 \mathrm{~T}$ are shown in Fig. 5 for tracks perpendicular to the pad row. In the calculation the value of the diffusion constant $(D)$ given by MAGBOLTZ was used. The figure tells us that under a strong magnetic field it is important to reduce the pad-pitch dominant region (at small drift distances) in the ILC-TPC by enhancing the charge sharing among the readout pads, in order to maintain good resolution over the entire sensitive volume.

In this respect GEM readout is favorable since electrons are significantly diffused (defocused) after the gas amplification in the transfer and induction gaps. The amount of diffusion can be optimized by adjusting the gap widths. It is worth noting here that the suppression of transverse diffusion in the transfer and induction gaps due to a magnetic field is in general significantly weaker than that in the drift region because of the shorter mean free time of drift electrons under higher electric fields. ${ }^{6}$

The combination of MicroMEGAS (or GEMs) and a resistive anode plane is also a good candidate for the detection device since it makes the effective pad pitch smaller [8]. Another ambitious possibility is the pixel readout (digital TPC) [9], in which the physical size of the readout elements is very small.

\footnotetext{
${ }^{5}$ It should be noted that PRF is assumed to be static in the analytic calculation [5], whereas it is stochastic in reality since a finite number of electrons are involved in the generation of pad signals.

${ }^{6}$ In presence of an axial magnetic field $(B)$ the transverse diffusion constant is given by $D(B=0) / \sqrt{1+\omega^{2} \tau^{2}}$, where $\omega \equiv e B / m$, the electron cyclotron frequency, and $\tau$ is the mean free time of drift electrons.
} 


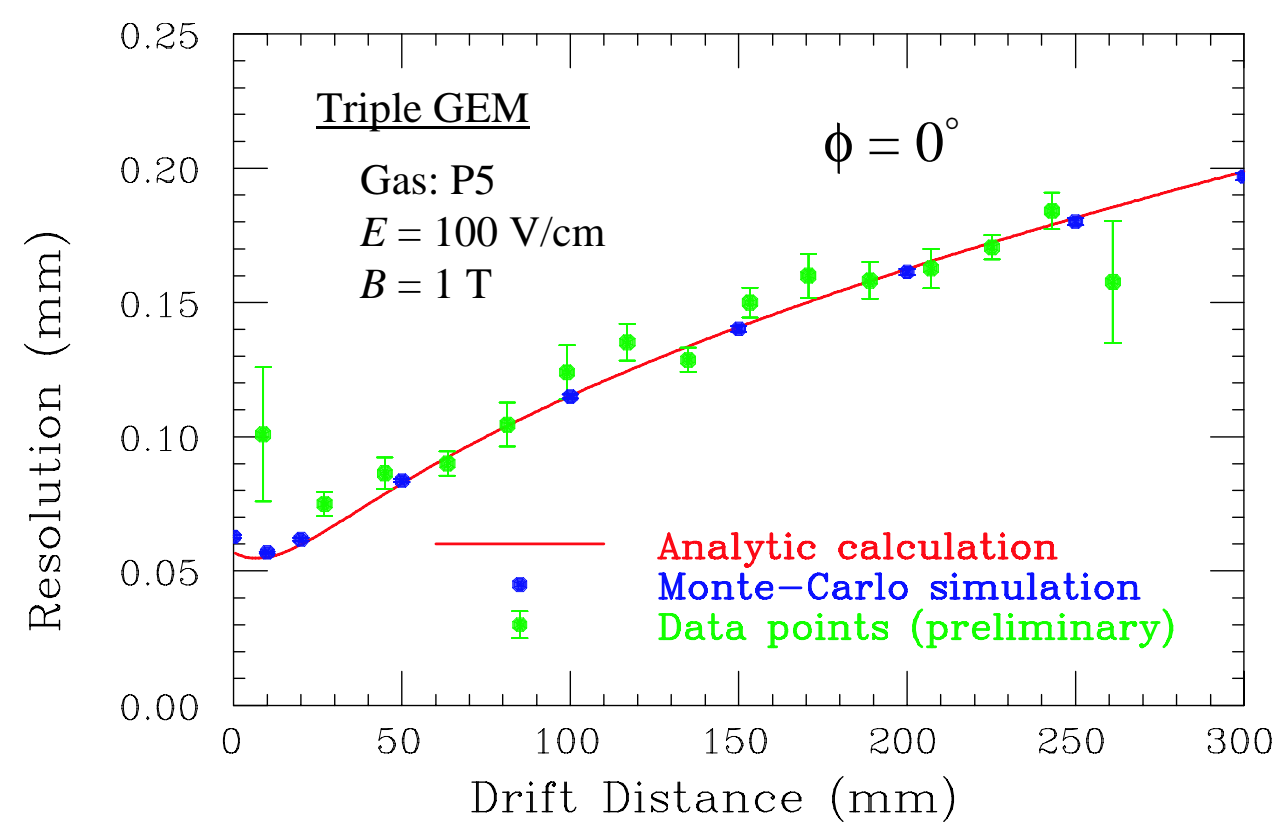

Figure 4: Comparison between the analytic calculation and the Monte-Carlo simulation of the spatial resolution. In the calculation $N_{\text {eff }}$ is assumed to be 22 and PRF is assumed to be a Gaussian with $\sigma=363 \mu \mathrm{m}$. In the simulation the relative variance of the avalanche fluctuation is supposed to be $2 / 3$ for each GEM. The diffusion constant $(D)$ is set to $166 \mu \mathrm{m} / \sqrt{\mathrm{cm}}$ in both cases.

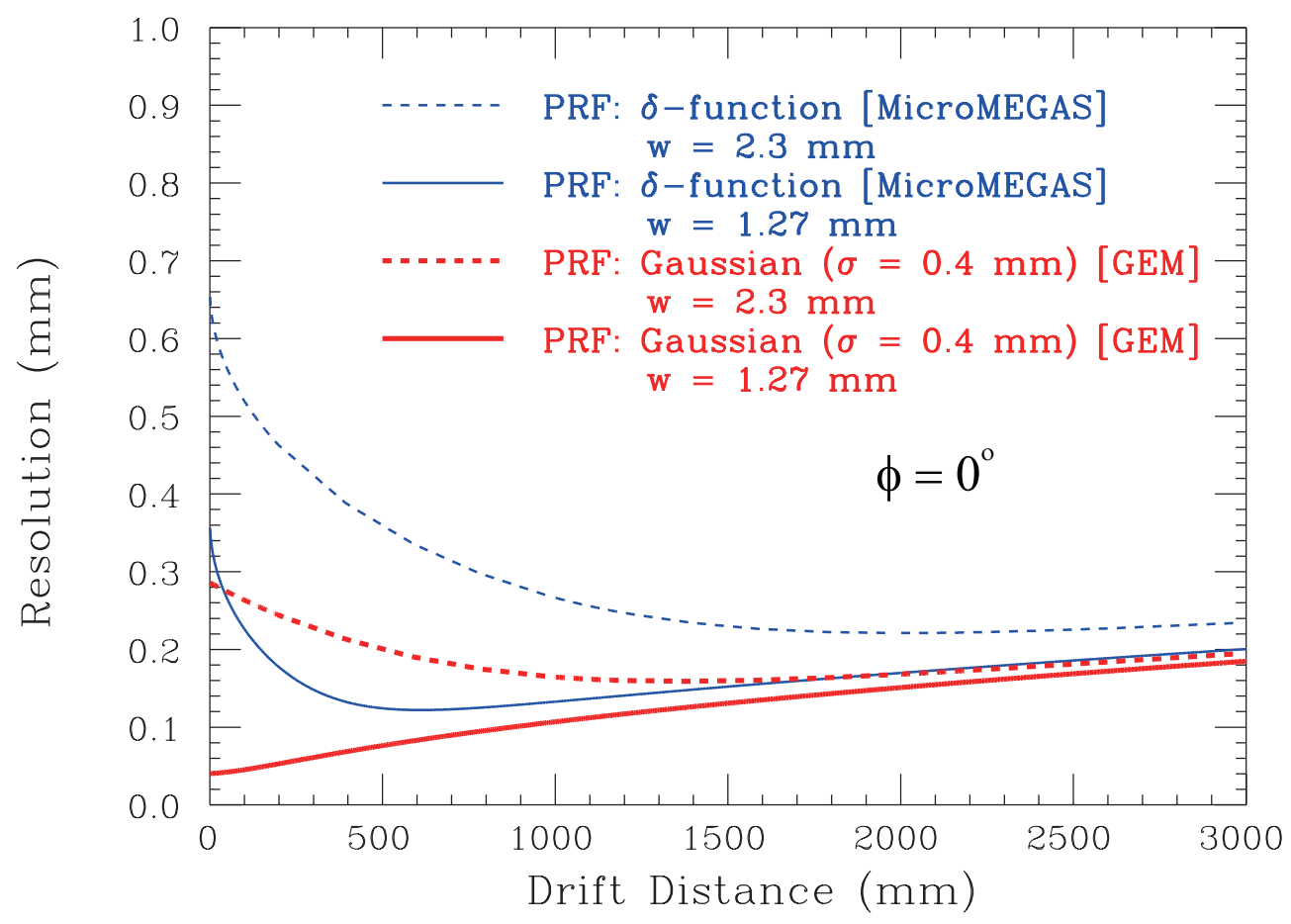

Figure 5: Expected spatial resolution of the ILC-TPC obtained with MicroMEGAS or GEMs. Gas: Ar-methane (5\%), $E=100 \mathrm{~V} / \mathrm{cm}, B=4 \mathrm{~T}(D=50 \mu \mathrm{m} / \sqrt{\mathrm{cm}})$, and $N_{\text {eff }}=22$. 


\section{Conclusions}

The prototype TPC equipped with a MicroMEGAS or with GEMs operated stably during the beam tests. The tests provided us with an insight into the spatial resolution along the pad row direction, which is achievable with a TPC equipped with an MPGD readout.

- The obtained spatial resolution is understood in terms of pad pitch, pad response function (PRF), diffusion constant, and the effective number of electrons.

- The expected resolution can be estimated by a numerical calculation based on a simple analytic formula, easy to code and fast, though it is applicable only to tracks perpendicular to the pad row direction.

- In the case of MicroMEGAS, the spatial resolution as a function of the drift distance is well described by the analytic formula, assuming a $\delta$ function for PRF.

- In the case of GEMs, the spatial resolution as a function of the drift distance is satisfactorily described by the analytic formula, assuming a Gaussian for PRF, with its width determined from the intercept of the pad-response width squared as a function of the drift distance.

- It is important to make the pad pitch small, physically or effectively, in order to reduce both the overall offset term $\left(\sigma_{\mathrm{X} 0}\right)$ at long drift distances and the resolution degradation due to the finite pad pitch at small drift distances.

- The spatial resolution required for the ILC-TPC $(\leq 200 \mu \mathrm{m}$ for the maximum drift length of $\sim 2.5 \mathrm{~m}$ ) is now within reach for tracks perpendicular to the pad row, with a standard GEM readout.

\section{Acknowledgements}

We are grateful to the people at KEK cryogenics science center and the members of IPNS cryogenic group for the preparation and the operation of the superconducting magnet. One of the authors (M. K.) would like to thank the organizers of the 11th Vienna Conference on Instrumentation for their support and hospitality. He is also grateful to many colleagues of the ILC-TPC collaboration for their continuous encouragement and support, and for fruitful discussions.

\section{References}

[1] TESLA Technical Design Report, DESY 2001-011.

[2] GLC Project Report, KEK Report 2003-7.

[3] Y. Giomataris, et al., Nucl. Instr. and Meth. A 376 (1996) 29.

[4] F. Sauli, Nucl. Instr. and Meth. A 386 (1997) 531.

[5] M. Kobayashi, et al., KEK Preprint 2006-35.

[6] M. Kobayashi, Nucl. Instr. and Meth. A 562 (2006) 136.

[7] S.F. Biagi, Nucl. Instr. and Meth. A 421 (1999) 234.

[8] M.S. Dixit, et al., Nucl. Instr. and Meth. A 518 (2004) 721.

[9] P. Colas, et al., Nucl. Instr. and Meth. A 535 (2004) 506. 